\title{
Piezoelectric properties of twinned ferroelectric perovskites with head-to-head and tail-to-tail domain walls
}

\author{
P. Ondrejkovic, ${ }^{1}$ P. Marton, ${ }^{1}$ M. Guennou, ${ }^{1}$ N. Setter, ${ }^{2}$ and J. Hlinka ${ }^{1}$ \\ ${ }^{1}$ Institute of Physics, Academy of Sciences of the Czech Republic, Na Slovance 2, 18221 Praha 8, Czech Republic \\ ${ }^{2}$ Ceramics Laboratory, Swiss Federal Institute of Technology, Lausanne CH-1015, Switzerland
}

(Received 13 May 2013; published 22 July 2013)

\begin{abstract}
Longitudinal piezoelectric coefficient of a twinned ferroelectric perovskite material with an array of partially compensated head-to-head and tail-to-tail 90-degree domain walls has been studied by phase-field simulations in the framework of the Ginzburg-Landau-Devonshire model of $\mathrm{BaTiO}_{3}$ ferroelectric. In particular, it is shown that the magnitude of the build-in extrinsic charge at the domain wall and the nanoscale domain size can both promote rotation of the static polarization vector within the body of adjacent domains. This polarization rotation drives the domain closer to an orthorhombic state, and the proximity to this ferroelectric-ferroelectric phase transition is directly responsible for the enhancement of the properties. Our simulations and the theory also suggest that the same system with nominally overcompensated charged walls may show a negative effective longitudinal piezoelectric coefficient. The obtained results can be used for quantitative estimates of piezoelectric properties of domain-engineered crystals.
\end{abstract}

DOI: 10.1103/PhysRevB.88.024114

PACS number(s): 77.80.Dj, 77.84.-s

\section{INTRODUCTION}

A wide range of practical instruments exploits the excellent piezoelectric properties of perovskite ferroelectrics. It is believed that the organization and the density of non-180degree domain walls in these materials has a sizable impact on their electromechanical properties. ${ }^{1-4}$ In principle, this gives a considerable opportunity for tailoring piezoelectric properties of a given ferroelectric material by modification of its domain structure. ${ }^{5}$ With this perspective, the macroscopic piezoelectric response of the basic plausible domain configurations has been already analyzed by various theoretical approaches. In particular, the laminate domain structures in the tetragonal ferroelectric phase have been analyzed in the framework of effective-medium theories based on the analysis of the electromechanical matching of homogeneous domains, ${ }^{6,7}$ but also in the framework of phase-field models allowing to treat size-dependent effects. ${ }^{4,8,9}$ Several recent works have drawn attention to the extraordinary possibilities of incorporating the so-called head-to-head (HH) and tail-totail (TT) domain walls. ${ }^{9-13}$ These domain walls have been observed in real materials but they are nominally charged so that their existence requires some charge compensation mechanism. Since ferroelectric perovskites can be usually considered as wide-band semiconductors, it seems likely that under convenient conditions, the compensation could be provided directly through the charge carriers from valence or conduction bands, for example, due to the band-bending mechanism discussed recently in Refs. 10-12.

Another possibility, advocated in Ref. 13, suggests that the compensation could be provided by fixed extrinsic charges related to some ionic defects, such as oxygen or even cationic vacancies in $\mathrm{ABO}_{3}$ perovskite crystal lattice, segregated at the domain wall. Interestingly, results of Ref. 13 suggest that the piezoelectric properties could be considerably enhanced when the charge compensation by ionic defects is actually quite far from the ideal nominally required amount. The fact that $\mathrm{HH}$ and TT domain walls can survive in the lattice even when the compensating charge densities deviate from nominally required values is exploited also in this work. We consider that the degree of compensation can be modified in experiments by varying slightly the chemical composition and concentrations of available defects per domain wall area or by modifying the condition of the annealing process during which the ionic defects are likely to diffuse towards the charged domain walls. But we do not discuss this process here. Our aim is to provide direct insight to the enhancement of piezoelectric properties of the twinned $\mathrm{BaTiO}_{3}$, predicted in Ref. 13 by complementary analytical and phase-field simulations using the GinzburgLandau-Devonshire (GLD) model with the parameters of Refs. 4 and 14.

\section{TECHNICAL DETAILS}

Phase-field simulations presented in this article are based on the phenomenological GLD model ${ }^{4,15}$ in which the excess Gibbs free-energy functional $F$ is expressed in terms of lowest-order polynomials of ferroelectric polarization $P_{i}$, its spatial derivatives $P_{i, j}=\partial P_{i} / \partial x_{j}$ and strain components $e_{i j}=\left(\partial u_{i} / \partial x_{j}+\partial u_{j} / \partial x_{i}\right) / 2, i, j=1-3$ as

$$
F=\int d \mathbf{r} f_{\mathrm{GLD}}\left[\left\{P_{i}, P_{i, j}, e_{i j}\right\}\right]+F_{\mathrm{dip}}\left[\left\{P_{i}\right\}\right],
$$

where the first term

$$
\begin{aligned}
f_{\mathrm{GLD}} & {\left[\left\{P_{i}, P_{i, j}, e_{i j}\right\}\right] } \\
= & \alpha_{1} \sum_{i} P_{i}^{2}+\alpha_{11}^{(\mathrm{e})} \sum_{i} P_{i}^{4}+\alpha_{12}^{(\mathrm{e})} \sum_{i>j} P_{i}^{2} P_{j}^{2} \\
& +\alpha_{111} \sum_{i} P_{i}^{6}+\alpha_{112} \sum_{i>j}\left(P_{i}^{4} P_{j}^{2}+P_{j}^{4} P_{i}^{2}\right) \\
& +\alpha_{123} P_{1}^{2} P_{2}^{2} P_{3}^{2}-P_{i} E_{i} \\
& +\frac{1}{2} C_{i j k l} e_{i j} e_{k l}-q_{i j k l} e_{i j} P_{k} P_{l}+\frac{1}{2} G_{i j k l} P_{i, j} P_{k, l}
\end{aligned}
$$


stands for the usual GLD functional. The greek letter symbols are parameters defining the fully clamped Landau-Ginzburg potential at a given temperature, $C_{i j k l}, q_{i j k l}$, and $G_{i j k l}$ stand for components of elastic, electrostriction, and gradient tensors, respectively, and $E_{i}$ stands for the component of applied electric field, which acts as an independent thermodynamical variable of the local Gibbs function. The other term in Eq. (1) describes the electrostatic energy ${ }^{16,17}$

$$
F_{\text {dip }}\left[\left\{P_{i}\right\}\right]=-\frac{1}{2} \int d \mathbf{r}\left[\mathbf{E}_{\mathrm{dip}}(\mathbf{r}) \cdot \mathbf{P}(\mathbf{r})\right],
$$

associated with the long-range interaction of individual dipoles with the electric fields of all other dipoles, expressed via the inhomogeneous depolarization field $\mathbf{E}_{\text {dip }}$ created by the inhomogeneous part of the field of polarization:

$$
\mathbf{E}_{\mathrm{dip}}(\mathbf{r})=\frac{-1}{4 \pi \epsilon_{0} \epsilon_{\mathrm{B}}} \int d \mathbf{r}^{\prime}\left\{\frac{\mathbf{P}\left(\mathbf{r}^{\prime}\right)}{|\mathbf{R}|^{3}}-\frac{3\left[\mathbf{P}\left(\mathbf{r}^{\prime}\right) \cdot \mathbf{R}\right] \mathbf{R}}{|\mathbf{R}|^{5}}\right\},
$$

where $\mathbf{R}=\mathbf{r}-\mathbf{r}^{\prime}, \epsilon_{\mathrm{B}}$ is the relative background permittivity of the medium (without the primary order parameter contribution), and $\epsilon_{0}$ is the permittivity of vacuum. Let us stress that in the present problem, the field of polarization is restricted by periodic boundary conditions, which implies that the long-range spatial average of the electric field $\mathbf{E}_{\text {dip }}$ generated by the field of polarization itself is vanishing.

In order to find the equilibrated domain structure under specified conditions, we have applied the usual phase-field approach ${ }^{16,18-21}$ consisting in a simulation of the natural "equilibration" process by numerical solution of the corresponding time-dependent Ginzburg-Landau equation (TDGL) for the field of polarization:

$$
\frac{\partial P_{i}}{\partial t}=-\Gamma \frac{\delta F}{\delta P_{i}}
$$

where $\Gamma$ is a kinetic coefficient controlling the energy dissipation rate of the system. Mechanical equilibrium is assumed to be achieved at each instant so that the inhomogeneous strain field can be eliminated from the energy functional of Eq. (1) using the corresponding Euler-Lagrange equations. ${ }^{17-19}$ The homogeneous average strain was evaluated in a similar way assuming fixed external stress boundary conditions.

We used phenomenological parameters for roomtemperature $\mathrm{BaTiO}_{3}$ that were selected as follows: ${ }^{4,15} \alpha_{1}=$ $-2.77 \times 10^{7} \mathrm{JmC}^{-2}, \alpha_{11}^{(\mathrm{e})}=1.701 \times 10^{8} \mathrm{Jm}^{5} \mathrm{C}^{-4}, \alpha_{12}^{(\mathrm{e})}=$ $-2.760 \times 10^{8} \mathrm{Jm}^{5} \mathrm{C}^{-4}, \alpha_{111}=8.004 \times 10^{9} \mathrm{Jm}^{9} \mathrm{C}^{-6}, \alpha_{112}=$ $4.470 \times 10^{9} \mathrm{Jm}^{9} \mathrm{C}^{-6}, \alpha_{123}=4.910 \times 10^{9} \mathrm{Jm}^{9} \mathrm{C}^{-6}, \mathrm{G}_{11}=$ $51 \times 10^{-11} \mathrm{Jm}^{3} \mathrm{C}^{-2}, \mathrm{G}_{12}=-2 \times 10^{-11} \mathrm{Jm}^{3} \mathrm{C}^{-2}, \mathrm{G}_{44}=$ $2 \times 10^{-11} \mathrm{Jm}^{3} \mathrm{C}^{-2}, \mathrm{q}_{11}=14.20 \times 10^{9} \mathrm{JmC}^{-2}, \mathrm{q}_{12}=$ $-0.74 \times 10^{9} \mathrm{JmC}^{-2}, \mathrm{q}_{44}=3.14 \times 10^{9} \mathrm{JmC}^{-2}, \mathrm{C}_{11}=27.50 \times$ $10^{10} \mathrm{Jm}^{-3}, \mathrm{C}_{12}=17.90 \times 10^{10} \mathrm{Jm}^{-3}, \mathrm{C}_{44}=5.43 \times 10^{10}$ $\mathrm{Jm}^{-3}$, and $\epsilon_{\mathrm{B}}=7.35$. These parameters are the same as those defined as "model I" (at $298 \mathrm{~K}$ ) in Ref. 15, except for the parameter $q_{44}$, which has been later corrected by a factor of two ${ }^{4}$. We use a kinetic coefficient $\Gamma=4 \times 10^{4} \mathrm{C}^{2} \mathrm{~J}^{-1} \mathrm{~m}^{-1} \mathrm{~s}^{-1}$ to define realistic time scales in the simulated processes, ${ }^{22}$ but this choice has no effect on the results presented here. Note that the experimental uncertainty of these parameters is obviously far beyond the numerical precision given here. Nevertheless, we prefer to provide here the actual values used in the calculation as this might be of use to those willing to reproduce our results. The same holds for most of the derived quantities given in this paper.

The inherent symmetry of the problem treated in this article allowed to perform the simulation within a $2 \mathrm{D}$ rectangular discrete array (typically, $128 \times 128$ individual sites), fulfilling periodic boundary conditions. Equation (5) was resolved numerically in Fourier space by a second-order semi-implicit method with spatial steps $0.5 \mathrm{~nm}$ and individual time steps $2 \mathrm{fs}$.

The piezoelectric properties of a given domain configuration were derived by a straightforward procedure: at first, the initial structure was relaxed (equilibrated) for about 50-100 ps at $T=298 \mathrm{~K}$. Then, the relaxation was repeated with that equilibrated structure under a small applied probing homogeneous electric field. The average of the induced strain was evaluated for several values of the probing field. Then, the average strain as a function of the probing field was fitted to a polynomial function and the linear increment in average strain was determined. The quadratic increment describing the electrostriction contribution was negligible for the small probing fields applied here. Finally, the ratio of the linear increment and the probing field was used to evaluate the converse (indirect) piezoelectric coefficient. Similar equilibrating under small homogeneous steplike applied stress was used to determine the direct piezoelectric coefficient.

\section{INVESTIGATED DOMAIN STRUCTURE}

In this study, we have investigated the room-temperature piezoelectric response of domain-engineered $\mathrm{BaTiO}_{3}$ crystal assuming the particular case of the domain structure sketched in Fig. 1(a). This domain structure was stabilized by a piecewise homogeneous macroscopic field sketched in Fig. 1(b). What is the motivation for this choice? Within the GLD model described above, the lowest energy state of an infinite sample at ambient stress-free conditions corresponds to the single-domain tetragonal phase with spontaneous polarization $\mathbf{P}_{\mathrm{S}}, P_{\mathrm{S}}=\left|\mathbf{P}_{\mathrm{S}}\right|=0.2652 \mathrm{Cm}^{-2}$. When such an ideal domain state is cut by a plane with an outward pointing normal $\mathbf{n}$, such surface possesses (an uncompensated) surface bound charge with planar density $\sigma_{\mathrm{S}}=\mathbf{P}_{\mathrm{S}} \cdot \mathbf{n}$. Let us consider a piece-wise homogeneous polarization configuration shown in Fig. 1(a) (equivalent to those considered in Ref. 13). It is composed of alternating (100) and $(0 \overline{1} 0)$ oriented tetragonal ferroelectric domain states of equal thickness separated by infinitely sharp $90^{\circ}$ domain walls, perpendicular to the
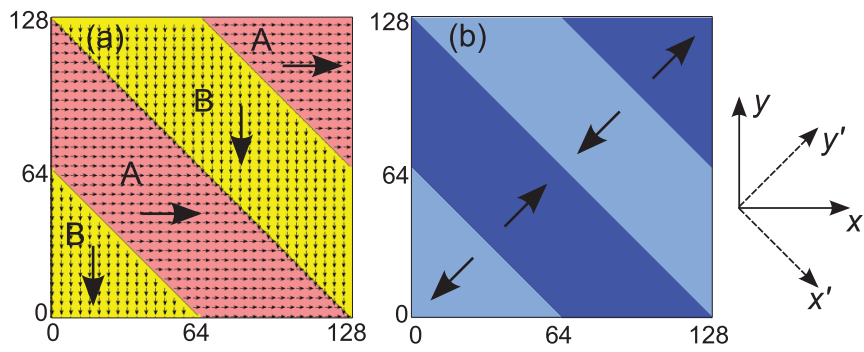

FIG. 1. (Color online) Schematic representation of initial conditions: (a) simple two-dimensional lamellar domain structure with charged $90^{\circ}$ domain walls assumed in present simulations and (b) the imposed piece-wise homogenous electric field $\mathbf{E}_{\sigma}$, associated with the compensating charge distribution defined by Eq. (7). 
direction $\mathbf{y}^{\prime}=(1,1,0) / \sqrt{2}$. Because of the alternating $\mathrm{HH}$ and TT domain wall arrangement, these domain boundaries possess planar charge densities $\sigma_{\text {dep }}=2 \sigma_{\mathrm{S}}= \pm 2\left|\mathbf{P}_{\mathrm{S}} \cdot \mathbf{y}^{\prime}\right|(\mathrm{HH}$ domain wall is positively charged). In a material with a background permittivity $\epsilon_{\mathrm{B}}$, this charge distribution would create an inhomogeneous depolarization field

$$
\mathbf{E}_{\mathrm{dep}}=-\frac{\left(\mathbf{P}_{\mathrm{S}} \cdot \mathbf{y}^{\prime}\right) \mathbf{y}^{\prime}}{\epsilon_{\mathrm{B}} \epsilon_{0}} .
$$

It is well known that in the absence of additional chargecompensation mechanisms, such a huge depolarization field (about $2.7 \mathrm{GV} / \mathrm{m}$ in the room-temperature $\mathrm{BaTiO}_{3}$ ) would destroy the assumed domain structure, and indeed, it is what happens when the structure of Fig. 1(a) is used as an initial configuration in phase-field simulations.

As already mentioned, plausible carrier species involved in the charge compensation of the bound charge density $\sigma_{\mathrm{dep}}$ are either the highly mobile electrons and holes available at domain walls due to electronic band-bending mechanism, ${ }^{10-12}$ or much less mobile ionic defects segregated at these domain walls, such as the oxygen or cationic vacancies or other charged defects. ${ }^{13}$ While the electronic subsystem could in principle follow the piezoelectric response, the compensation ionic charge density can be regarded as a fixed static charge distribution. For simplicity, we shall consider the latter case here, even though with some caution, the present results can be also used for gaining some additional insight into the former case as well (e.g., in combination with the analysis of Ref. 12).

It has been shown ${ }^{13}$ that the HH and TT walls may survive even when the magnitude of the imposed compensation charge density considerably differs from $\sigma_{\text {dep }}$. Therefore we shall express the imposed compensation density as

$$
\sigma=-\rho^{*} \sigma_{\mathrm{S}}=-\frac{1}{2} \rho^{*} \sigma_{\mathrm{dep}} .
$$

Here we have used, along with the Ref. 13, a dimensionless charge density parameter $\rho^{*}$, indicating the imposed compensation density in units of $P_{\mathrm{S}} / \sqrt{2}$. For simplicity, the imposed compensation charge density is assumed to be of the same magnitude for both HH and TT walls. The inhomogeneous electric field $\mathbf{E}_{\sigma}$ caused by the imposed compensation charge density $\sigma$ is directed opposite to the nominal depolarization field $\mathbf{E}_{\mathrm{dep}}$, and it can be expressed as $\mathbf{E}_{\sigma}=-\frac{1}{2} \rho^{*} \mathbf{E}_{\mathrm{dep}}$ [see Fig. 1(b)]. Therefore the exact compensation of the nominal bound charge density $\sigma_{\text {dep }}$ and of the nominal $\mathbf{E}_{\text {dep }}$ takes place for $\rho^{*}=2$. Therefore the electric field $\mathbf{E}$ of Eq. (2) applied in the present phase-field simulations consists of (i) zero or small homogenous probing field and (ii) a rather strong, piece-wise homogeneous electric field $\mathbf{E}_{\text {dep }}$, describing the effect of the imposed charge carriers.

Obviously, in our phase-field simulations, polarization is allowed to evolve under TDGL equation (5), so that the domain walls have finite thicknesses. Moreover, the polarization within the body of ferroelectric domains differs from spontaneous single domain value of $\mathbf{P}_{\mathrm{S}}$ due to the influence of the following three aspects: (i) the very presence of domain walls, (ii) the domain wall distance (domain size effect), and (iii) the imposed fixed compensation charge density $\sigma$. Consequently, the actual depolarization field due to the equilibrium polarization distribution can be considerably different from the nominal value of Eq. (6). As we shall see, the field of polarization can, in fact, adjust to a broad range of values of $\rho^{*}$ without destroying the basic pattern of $\mathrm{HH}$ and TT domain wall array.

\section{RESULTS}

The model for $\mathrm{BaTiO}_{3}$ that we used in our previous works $^{4,14}$ is somewhat different from the one used in Ref. 13, and so it is interesting to compare the predictions of both models for an equivalent domain structure. For this purpose, we have chosen the set of data obtained for the domain arrangement shown in Fig. 1(a) with the 22.6-nm-thick domains. The comparison of the data obtained for the longitudinal piezoelectric coefficient $d_{33}^{[100]}$ is shown in Fig. 2. In both cases, data show a dramatic increase in the response when $\rho^{*}$ is significantly smaller than 2 . Moreover, we have extended the simulations also to the case when the $\rho^{*}$ parameter is significantly larger than 2 . In this case, a marked dependence is also observed, but very interestingly, the material then shows a negative longitudinal piezoelectric coefficient $d_{33}^{[100]}$.

All data indicated in Fig. 2 were obtained as a converse piezoelectric response, i.e., from linear expansion of the material in the [100] direction in response to a small homogeneous probing bias electric field $(100 \mathrm{~V} / \mathrm{m})$ in the same direction. We have checked that the effect was linear in the whole investigated range of parameters $\rho^{*}$ (explicit electrostriction contribution was negligible). In addition, we also simulated the direct piezoelectric effect (polarization induced by a small uniaxial stress applied in the [100] direction) and obtained same values.

One of the obvious advantages of the phase-field simulations is that the calculation can automatically take into account the finite size effects related to the polarization correlation lengths (natural domain wall thickness). Therefore we have also investigated the influence of the domain size on the

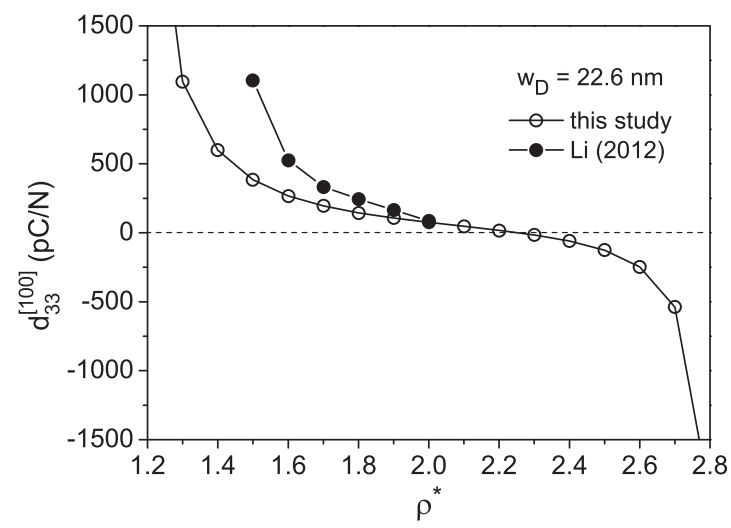

FIG. 2. Effective longitudinal piezoelectric coefficient $d_{33}^{[100]}$ of the $\mathrm{BaTiO}_{3}$ ferroelectric material with a hypothetic domain structure sketched in Fig. 1(a) as a function of the compensation charge parameter $\rho^{*}$ (for 22.6-nm-thick domains). Values obtained from the present phase-field calculations (open circles) can be compared to the values of the phase-field calculations given in Table III of Ref. 13 (full circles). In both cases, the values were determined from the overall elongation of the material in the [100] direction in response to a small probing electric field in the same direction (simulation of a converse piezoelectric response). 


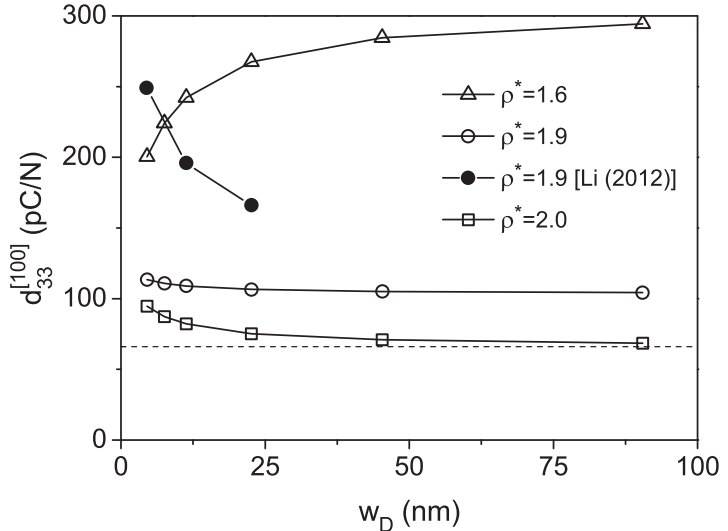

FIG. 3. Effective longitudinal piezoelectric coefficients $d_{33}^{[100]}$ as a function of domain wall distance $w_{\mathrm{D}}$ for selected values of compensation charge parameter $\rho^{*}$. Open point symbols refer to this study, full circles are data from Table IV of Ref. 13. Full lines are guides for eye, the dotted line indicates the analytically calculated limiting value of the $d_{33}^{[100]}$ piezoelectric coefficient for $\rho^{*}=2$ case (see in Sec. V A).

macroscopic piezoelectric response of our system. Similarly, as in Ref. 13, we have found that decreasing domain size can markedly enhance the piezoelectric properties (see Fig. 3). At the same time, we have found a much smaller effect in the reported case of $\rho^{*}=1.9$ (see full and open point symbols in Fig. 3). We have noticed that a comparable or even greater enhancement can be still achieved by decreasing $\rho^{*}$ rather than the domain wall distance, what could actually turn out to be more feasible in experiments (compare the open circle and open triangle symbols in Fig. 3). Surprisingly, we have observed that the longitudinal piezoelectric coefficient $d_{33}^{[100]}$ can also decrease with decreasing domain size (e.g., for $\rho^{*}=1.6$, see open triangle symbols in Fig. 3).

In order to get more insight into the mechanism of these effects, it is interesting to explore the local contributions to the calculated piezoelectric response. For this purpose, we have analyzed the local change of the field of polarization induced by a small homogeneous probing uniaxial stress along the [100] direction $\left(\sigma_{x x}=10^{4} \mathrm{~Pa}\right.$, other stress tensor components were zero). Local polarization changes along [100] and [010] directions $\left(\delta P_{x}\right.$ and $\left.\delta P_{y}\right)$ in the vicinity of the $\mathrm{HH}$ domain wall are shown in Figs. 4(a) and 4(b), respectively. Obviously, the longitudinal piezoelectric coefficient $d_{33}^{[100]}=d_{x x x}$ can be evaluated as the spatial average of $\delta P_{x}$ over the whole specimen, divided by the applied stress $\sigma_{x x}$. One can thus easily see from Fig. 4(a) that the huge sensitivity of the longitudinal piezoelectric coefficient is related to changes in the B domain, i.e., in the domain having its nominal spontaneous polarization perpendicular to the applied stress.

Let us stress that the calculated domain walls (i.e., kinks in the spatial distribution of the ferroelectric polarization) are not a priori fixed in our simulations but they are strongly attracted to the rigidly fixed build-in charge layers. Therefore domain wall displacements under the probing fields are negligibly small. It could be also understood from Fig. 4, which shows that the contribution to the piezoelectric response is not
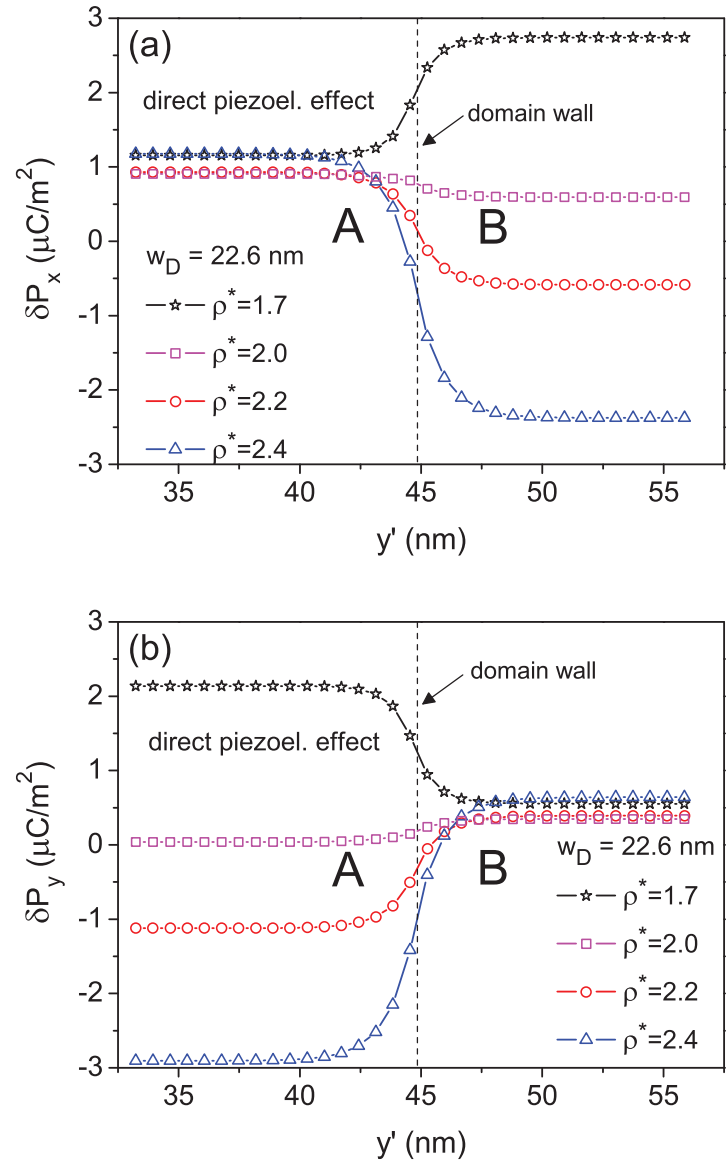

FIG. 4. (Color online) Local polarization increment induced by a small, $x$-axis tensile uniaxial stress $\left(\sigma_{x x}=10^{4} \mathrm{~Pa}\right)$, traced along the $y^{\prime}$ direction in the vicinity of a $\mathrm{HH}$ domain wall. The domain wall distance is fixed to $22.6 \mathrm{~nm}$, various symbols refer to data calculated for various values of the charge parameter $\rho^{*}$, (a) $x$ component of the polarization increment and (b) $y$ component of the polarization increment.

significantly enhanced in the domain wall area. In this sense, domain walls in our simulations may be considered as pinned.

Previously, authors of Ref. 13 proposed that the decrease of the piezoelectric response with $\rho^{*}$ approaching the value of 2 (see Fig. 2) might be caused by enhanced domain wall pinning. However, our results clearly show that pinning is very strong for all investigated values of $\rho^{*}$. In reality, our Fig. 4 testifies that this tendency apparent from Fig. 2 is rather caused by variation of the piezoelectric response within the domain interior. The reason of this variation will be discussed in the next section.

\section{DISCUSSION}

A. Nominally compensated walls $\left(\rho^{*}=2\right)$.

Let us first discuss the $\rho^{*}=2$ ideal case when the amount of the imposed compensation charge exactly cancels the nominal, bulk depolarization field given by Eq. (6). In the limit of thick enough domains, the body of the domain is in the normal bulk state, and it can be described by its usual (equilibrium single-domain) material tensors. In this case, the effective macroscopic piezoelectric response of the multidomain state 
depends only on these material tensors. The resulting effective values obviously also involve the domain fraction ratio and the orientation of the domain wall interfaces, as they impose the classical boundary conditions for otherwise piecewise homogeneous electrical and mechanical fields. Convenient formalisms for the calculation of the effective piezoelectric response of such an ideal laminar domain structure have been considered previously by several authors (see Refs. 6,7,23 and 24 and earlier works cited there).

The effective piezoelectric tensor for the present domain structure [shown in Fig. 1(a), with equal domain fractions] can be most conveniently expressed in the Cartesian system $x^{\prime}, y^{\prime}, z$, obtained from the natural crystallographic $x, y, z$ system by $45^{\circ}$ rotation around the $z$ axis (see Fig. 1). The explicit formulas for the effective piezoelectric tensor components $d_{i j}^{\text {eff }} \operatorname{read}^{7}$

$$
\begin{aligned}
d_{11}^{\text {eff }}= & d_{11}^{\prime}-d_{12}^{\prime} \frac{\epsilon_{12}^{\prime}}{\epsilon_{22}^{\prime}}, \\
d_{12}^{\text {eff }}= & d_{12}^{\prime}-d_{22}^{\prime} \frac{\epsilon_{12}^{\prime}}{\epsilon_{22}^{\prime}}, \\
d_{13}^{\text {eff }}= & d_{13}^{\prime}-d_{23}^{\prime} \frac{\epsilon_{12}^{\prime}}{\epsilon_{22}^{\prime}}, \\
d_{35}^{\text {eff }}= & d_{35}^{\prime}, \quad \\
d_{26}^{\prime \mathrm{eff}}= & d_{26}^{\prime}-\frac{s_{36}^{\prime}\left(d_{23}^{\prime} s_{11}^{\prime}-d_{12}^{\prime} s_{13}^{\prime}\right)}{s_{33}^{\prime} s_{11}^{\prime}-s_{13}^{\prime 2}} \\
& -\frac{s_{16}^{\prime}\left(-d_{23}^{\prime} s_{13}^{\prime}+d_{12}^{\prime} s_{33}^{\prime}\right)}{s_{33}^{\prime} s_{11}^{\prime}-s_{13}^{\prime 2}},
\end{aligned}
$$

where $d_{i j}^{\prime}, s_{i j}^{\prime}$, and $\epsilon_{i j}^{\prime}$ are single-domain material tensor components in the $x^{\prime}, y^{\prime}, z$ reference frame. ${ }^{7}$ From these expressions, and using the known stress-free room-temperature single-domain $\mathrm{BaTiO}_{3}$ material tensors corresponding to our GLD potential expansion in the conventional axis setting $\quad\left(\epsilon_{11}=3300, \epsilon_{33}=193, d_{31}=-40.9 \mathrm{pC} / \mathrm{N}, d_{33}=\right.$ $100 \mathrm{pC} / \mathrm{N}, d_{15}=448 \mathrm{pC} / \mathrm{N}, s_{11}=8.45 \times 10^{-3} \mathrm{GPa}^{-1}, s_{12}=$ $-1.97 \times 10^{-3} \mathrm{GPa}^{-1}, s_{13}=-5.34 \times 10^{-3} \mathrm{GPa}^{-1}, s_{33}=$ $13.33 \times 10^{-3} \mathrm{GPa}^{-1}, s_{44}=25.28 \times 10^{-3} \mathrm{GPa}^{-1}$, and $s_{66}=$ $18.42 \times 10^{-3} \mathrm{GPa}^{-1}$ ), we arrived at the following values of the effective piezoelectric tensor components: $d_{11}^{\text {eff }}=56.9 \mathrm{pC} / \mathrm{N}$, $d_{12}^{\text {eff }}=22.0 \mathrm{pC} / \mathrm{N}, d_{13}^{\text {eff }}=-54.6 \mathrm{pC} / \mathrm{N}, d_{35}^{\text {'eff }}=316.7 \mathrm{pC} / \mathrm{N}$, and $d_{26}^{\text {eff }}=107.6 \mathrm{pC} / \mathrm{N}$. Finally, the effective piezoelectric tensor was expressed in the original crystallographic frame $x, y, z$. The resulting values are displayed in the last column of Table I. For the sake of comparison, the full macroscopic piezoelectric tensor was also determined from phase-field simulations for $w_{\mathrm{D}}=22.6 \mathrm{~nm}$ and $w_{\mathrm{D}}=90.5 \mathrm{~nm}\left(\rho^{*}=2\right.$, see Table I). The agreement between the effective medium theory and the $w_{\mathrm{D}}=90.5 \mathrm{~nm}$ phase-field calculations is nice and suggests that, in this case, the 100-nm-thick domains could behave fairly much the same as the macroscopic domains.

Obviously, the piezoelectric component shown in the first row of Table I,

$$
d_{11}^{\mathrm{eff}}=\frac{1}{2 \sqrt{2}}\left(d_{11}^{\text {eff }}+d_{12}^{\text {eff }}+d_{26}^{\text {eff }}\right),
$$

TABLE I. Cartesian components of the macroscopic piezoelectric tensor (in the Voigt notation with respect to the natural pseudocubic system $x, y, z$ and in $\mathrm{pC} / \mathrm{N}$ units) of the multidomain structure shown in Fig. 1 with an ideal nominal compensation $\left(\rho^{*}=2\right)$. First and second numerical columns refer to data from phase-field simulations, the last column gives the prediction of the effective-medium theory relevant to the limit of thick domains.

\begin{tabular}{lccr}
\hline \hline & $w_{\mathrm{D}}=22.6 \mathrm{~nm}$ & $w_{\mathrm{D}}=90.5 \mathrm{~nm}$ & $w_{\mathrm{D}}=\infty$ \\
\hline$d_{11}^{\text {eff }}=-d_{22}^{\text {eff }}$ & 75.2 & 68.5 & 65.9 \\
$d_{12}^{\text {eff }}=-d_{21}^{\text {eff }}$ & -19.2 & -12.7 & -10.1 \\
$d_{13}^{\text {eff }}=-d_{23}^{\text {eff }}$ & -38.8 & -38.6 & -38.6 \\
$d_{16}^{\text {eff }}=-d_{26}^{\text {eff }}$ & -25.8 & -25.6 & -24.7 \\
$d_{34}^{\text {eff }}=-d_{35}^{\text {eff }}$ & -226.4 & -224.4 & -224.0 \\
\hline \hline
\end{tabular}

is identical to the so far discussed quantity $d_{33}^{[100]}$. Therefore the value from the last column was also included as the expected asymptotic "thick domain" value in Fig. 3. The data of Fig. 3 also reveal that the domain size has a rather small impact on the $d_{33}^{[100]}$ coefficient in the $\rho^{*}=2$ case. In fact, the size effect observed here is much smaller than the size effect predicted, e.g., for the uncharged head-to-tail domain-wall structures $\left(\rho^{*}=0\right)$ studied by us previously in Ref. 4 .

\section{B. Nominally uncompensated walls $\left(\rho^{*} \neq 2\right)$}

The present phase-field simulations imply that the most spectacular enhancement of the $d_{33}^{[100]}$ coefficient is achieved by a variation of the parameter $\rho^{*}\left(\rho^{*} \neq 2\right)$. Since the size effect seems to be a secondary effect, it can be expected that the piezoelectric enhancement is related to the modified response of the body of the domains, rather than to an unusual response of domain walls. Detailed inspection suggests that the variation of $\rho^{*}$ indeed induces a notable change of the equilibrium polarization vector in the body of the domain. This change can be roughly described as a polarization rotation around the $z$ axis. A similar rotation was present in previous studies of piezoelectric response of domain-engineered $\mathrm{BaTiO}_{3}{ }^{4,8,12}$ In fact, the general tendency to the polarization rotation in $\mathrm{BaTiO}_{3}$ and similar materials is known to be related to the large value of "transverse" permittivity element $\epsilon_{11}$ (i.e., $\epsilon_{11} / \epsilon_{33} \gg$ 1). ${ }^{2,12,25}$ What needs to be elucidated in this particular case is (i) the driving force inducing this static rotation and (ii) whether this rotation is directly involved in the enhancement of the considered piezoelectric coefficient.

In our case, the polarization vector of $\mathrm{A}$ and $\mathrm{B}$ domains tends to rotate in opposite sense (see Fig. 5). To quantify this effect, we have introduced angles $\alpha_{\mathrm{A}}$ and $\alpha_{\mathrm{B}}$, describing the deviation of the equilibrium polarization in the center of domains $\mathrm{A}$ and $\mathrm{B}$ from their nominal orientation [shown in Fig. 1(a)]. The $\alpha_{\mathrm{A}}$ and $\alpha_{\mathrm{B}}$ angles were read out from the simulated patterns and it was confirmed that they are equal for all the above considered cases [the angles were considered positive for the situation indicated in Fig. 5(b)], so that the domain structure maintains the original $C_{2 v}\left(m_{z} m_{y^{\prime}} 2_{x^{\prime}}\right)$ macroscopic symmetry. The equilibrium polarization is found to be tilted towards the domain wall interface for $\rho^{*}<2$ and away from it for $\rho^{*}>2$, respectively (see Fig. 5). This 

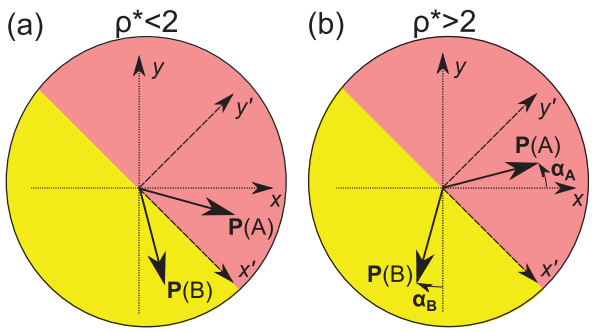

FIG. 5. (Color online) Schema indicating the observed tilt of the equilibrium polarization $\mathbf{P}(\mathrm{A})$ and $\mathbf{P}(\mathrm{B})$ in the middle of the domain $\mathrm{A}$ and $\mathrm{B}$, respectively. The equilibrium polarization is tilted towards the domain wall interface for $\rho^{*}<2$ as shown in (a) and away from it for $\rho^{*}>2$, as shown in (b). The role of the $\alpha_{\mathrm{A}}$ and $\alpha_{\mathrm{B}}$ angles is discussed in the main text.

tendency could be very easily understood: a positive angle implies enhancement of the normal component of polarization, and thus it also increases the depolarization field created by the mismatch of the normal components of the polarization in domains $\mathrm{A}$ and $\mathrm{B}$. This is precisely what has to be arranged to match the excess of the charge density $\left(\rho^{*}>2\right)$. Similarly, negative angles lead to smaller mismatch of the normal components of the polarization in domains $\mathrm{A}$ and $\mathrm{B}$, what is needed to accommodate the situations with small imposed compensation charge densities $\left(\rho^{*}<2\right)$.

The correlation of the $d_{33}^{[100]}$ piezoelectric coefficient with the tilt angle $\alpha_{\mathrm{A}}=\alpha_{\mathrm{B}}$ is apparent from Fig. 6. The values are somewhat spread by the dependence on the domain size, but it is clear that the overall dependence is strongly nonlinear and the most spectacular enhancement of the $d_{33}^{[100]}$ is observed for the $\alpha_{\mathrm{A}} \approx-20^{\circ}$, and the strongly negative $d_{33}^{[100]}$ values are obtained for $\alpha_{\mathrm{A}} \approx+20^{\circ}$. These critical polarization tilt angles correspond to the polarization pointing roughly in the middle between [100] and [110] crystallographic directions. It clearly demonstrates that the observed enhancement is related to the competition between tetragonal and orthorhombic ground states, inherent to the room-temperature GLD potential of $\mathrm{BaTiO}_{3}{ }^{4,12,26,27}$

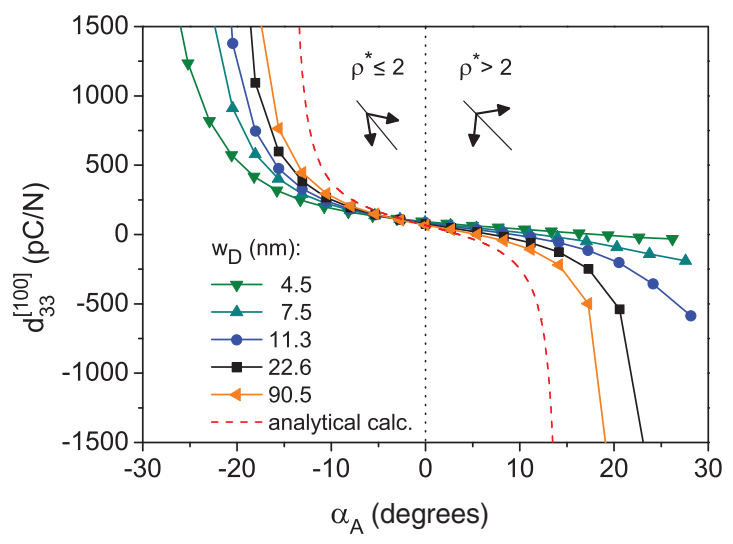

FIG. 6. (Color online) Effective longitudinal piezoelectric coefficients $d_{33}^{[100]}\left(w_{\mathrm{D}}, \rho^{*}\right)$ as a function of the deviation angle, joining the points with equal domain thicknesses. Dashed line was calculated from the Eqs. (15) and (22), derived in Sec. V C.

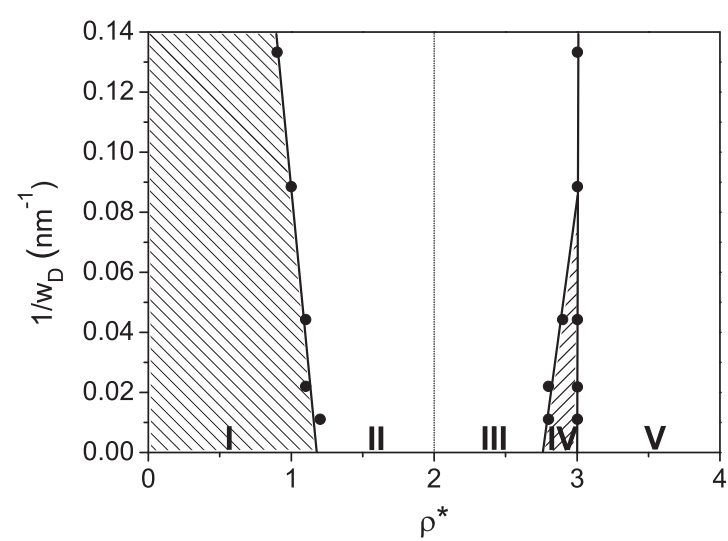

FIG. 7. Phase diagram showing stability domains of distinct types of domain structures obtained in present phase-field simulations for a range of parameters $w_{\mathrm{D}}$ and $\rho^{*}$, defining the domain wall distance and the imposed compensating charge density, respectively. Point symbols are determined from the divergence of the piezoelectric response as a function of $\rho^{*}$ (for selected fixed values of $w_{\mathrm{D}}$ ), lines are just guides for eye. Regions II and III can be considered as the original domain structure of Fig. 1(a), only with slight symmetric tilts of the polarization, as shown in Figs. 5(a) and 5(b), respectively. Shaded areas I and IV correspond to a lower-symmetry domain structure (monoclinic one), with unequal polarization tilts, sketched in Figs. 8(a) and 8(b). In the region V, the polarization is perpendicular to the imposed charge planes and the domain walls are transformed to 180-domain ones with $\mathrm{HH}$ and TT arrangements. See text for detailed explanation.

To shed additional light on this phenomenon, we have explored an even broader range of parameter $\rho^{*}$ and realized that for a certain range of parameters, the structure with $\alpha_{\mathrm{A}}=\alpha_{\mathrm{B}}$ is no more stable. The resulting stability map as a function of domain density $1 / w_{\mathrm{D}}$ and the extrinsic charge density parameter $\rho^{*}$ is sketched in Fig. 7. Indeed, only regions II and III correspond to the symmetric domain structure $\left(\alpha_{\mathrm{A}}=\alpha_{\mathrm{B}}\right)$ with domain states depicted in Figs. 5(a) and 5(b), respectively. In contrast, an asymmetric domain structure (with $\alpha_{\mathrm{A}} \neq \alpha_{\mathrm{B}}$ ) has lower energy than the symmetric one when $\rho^{*}$ and $w_{\mathrm{D}}$ parameters are kept within areas denoted as I and IV. Indeed, in region I, either the A- or B-domain state polarization is almost parallel to the domain wall, while in region IV, either the A or B-domain state polarization is almost perpendicular to the domain wall (see Fig. 8). In the region V, the structure
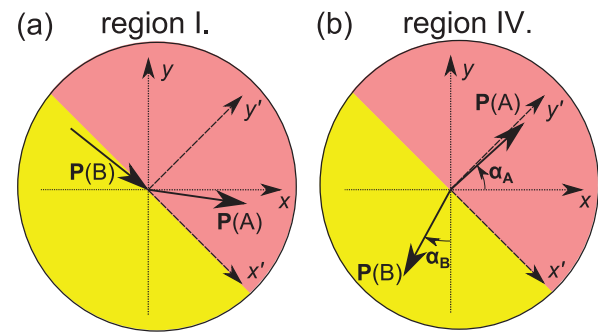

FIG. 8. (Color online) Schematic image indicating the observed tilt of the equilibrium polarization $\mathbf{P}(\mathrm{A})$ and $\mathbf{P}(\mathrm{B})$ in the middle of the domains $\mathrm{A}$ and $\mathrm{B}$, respectively, as obtained in our phase-field simulations with input parameters falling (a) in the region I and (b) in the region IV of Fig. 7. 
is again symmetric, but the polarization in both domains is perpendicular to the domain wall $\left(\alpha_{\mathrm{A}}=\alpha_{\mathrm{B}}=\pi / 4\right)$. It is easy to verify, that these geometrical arrangements provide the suitable step of the normal component of the polarization vector at the domain wall, needed for near-compensation for the imposed charge density $\rho^{*}$. In fact, we have seen that $[\mathbf{P}(A)-\mathbf{P}(B)] \cdot \mathbf{y}^{\prime}$ differs from $\rho^{*} P_{\mathrm{S}} / \sqrt{2}$ by less than $1 \%$.

It is obvious from Fig. 7. that the large magnitude of the piezoelectric response shown in Figs. 2 and 6 is associated with the borderline between regions I and II and the borderline between regions III and IV, respectively. The enhancement might be discussed even from the point of view of the Landau theory of symmetry breaking phase transition since at these borderlines, the domain structure is lowering its symmetry. It is worth noting that there is no truly single-domain orthorhombic state in our "phase diagram," since the imposed electric field due to the $\rho^{*}$ charge density induces a periodic wave that breaks the translation symmetry of the problem. In that sense, the structure is actually quite reminiscent of incommensurate dielectrics, where the enhancement of permittivity is also frequently found near various almost continuous transitions between modulated ferroelectric phases. ${ }^{28,29}$

In Fig. 6, data points calculated for equal domain wall sizes are connected with a common continuous line and plotted with the same symbols. Therefore one can see that there are systematic deviations related to the size effects. To some extent, the slope of the borderline between regions I and II in Fig. 7 allows to understand the spread of the data shown in Fig. 6. As could be guessed, the domain size effect is mainly caused by a partial charge compensation within the domain wall, which, obviously, plays a role only when domain sizes are of the order of the domain wall thickness.

\section{Mechanism of the piezoelectric enhancement}

Finally, it remains to understand how the polarization rotation influences the $d_{33}^{[100]}$ coefficient. For this purpose, we shall discuss only the regions II and III here. One can see from Fig. 4(a) that the strongest enhancement of the $d_{33}^{[100]}$ coefficient is originating from the response of the domain $\mathrm{B}$. Therefore let us investigate the properties of a homogenous domain state B under the bias depolarization field. For simplicity, we shall assume that this depolarization field results in a strict rotation of the spontaneous polarization by the angle $\alpha=\alpha_{\mathrm{B}}$ (its influence on the magnitude of the spontaneous polarization vector is neglected). Then, the spontaneous polarization can be written as $\mathbf{P}(B)=(-P \sin \alpha,-P \cos \alpha, 0)$. Under these assumptions, the permittivity tensor of domain $\mathrm{B}$ can be obtained from the Hessian of the stress-free Landau energy $f$,

$$
\epsilon_{i j}=\left(\frac{\partial^{2} f}{\partial P_{k} \partial P_{l}}\right)^{-1} .
$$

For a qualitative understanding, it is sufficient to keep only the lowest-order terms in $\alpha$. Recently, it has been explained that the static rotation of the spontaneous polarization greatly enhances the transverse susceptibility component $\epsilon_{x x}(\alpha) \cdot{ }^{12} \mathrm{~A}$ similar result is recovered here:

$$
\epsilon_{x x}(\alpha) \approx \frac{\epsilon_{11}}{1-K \alpha^{2}},
$$

with

$$
\begin{aligned}
K & =-\epsilon_{11} L+4 \epsilon_{11} \epsilon_{33} M^{2}, \\
L & =12 a_{11} P_{\mathrm{S}}^{2}-2 a_{12} P_{\mathrm{S}}^{2}+8 a_{112} P_{\mathrm{S}}^{4}, \\
M & =2 a_{12} P_{\mathrm{S}}^{2}+4 a_{112} P_{\mathrm{S}}^{4} .
\end{aligned}
$$

This formula allows to explain ${ }^{12}$ that for $K>0$ the permittivity $\epsilon_{x x}$ actually diverges as the angle approaches the critical value, where the denominator of the formula goes to zero. Using the adopted Landau-Devonshire expansion parameters given above and the stress-free Landau coefficients $a_{11}, a_{12}$ defined as ${ }^{15,26}$

$$
\begin{aligned}
& \alpha_{11}=\alpha_{11}^{(e)}-\frac{1}{6}\left(\frac{\hat{q}_{11}^{2}}{\hat{C}_{11}}+2 \frac{\hat{q}_{22}^{2}}{\hat{C}_{22}}\right), \\
& \alpha_{12}=\alpha_{12}^{(e)}-\frac{1}{6}\left(2 \frac{\hat{q}_{11}^{2}}{\hat{C}_{11}}-2 \frac{\hat{q}_{22}^{2}}{\hat{C}_{22}}+3 \frac{q_{44}^{2}}{C_{44}}\right),
\end{aligned}
$$

where

$$
\begin{aligned}
& \hat{C}_{11}=C_{11}+2 C_{12}, \quad \hat{C}_{12}=C_{11}-C_{12}, \\
& \hat{q}_{11}=q_{11}+2 q_{12}, \quad \hat{q}_{12}=q_{11}-q_{12},
\end{aligned}
$$

allows to calculate the parameters in the above equations as $K=15.7, L \doteq-4.15 \times 10^{8} \mathrm{~m} \mathrm{~F}^{-1}$, and $M \doteq 1.34 \times$ $10^{8} \mathrm{~m} \mathrm{~F}^{-1}$. In this case, the critical angle at which the denominator vanishes, $K \alpha_{\text {crit }}^{2} \approx 1$ is close to $15^{\circ}$, in a rough agreement with the divergence shown in Fig. 6. Obviously, the small value of this angle is directly related to the presence of subsidiary potential minima in Landau potential, associated with the metastable orthorhombic phase. ${ }^{12}$

This depolarization-field-induced divergence of the transversal susceptibility is responsible also for the enhancement of the $d_{33}^{[100]}$ piezoelectric coefficient. In the absence of external stresses, the equilibrium homogenous strain is determined by electrostriction coupling

$$
e_{x x}=Q_{11} P_{x}^{2}+Q_{12}\left(P_{y}^{2}+P_{z}^{2}\right),
$$

where $Q_{11}=0.11045 \mathrm{~m}^{4} \mathrm{C}^{-2}, Q_{12}=-0.0452 \mathrm{~m}^{4} \mathrm{C}^{-2}$ (see Ref. 15 and works cited therein). The longitudinal piezoelectric coupling along the direction $x$ (in domain state B) can be therefore expressed as

$$
d_{x x x}(\alpha)=\frac{\partial e_{x x}}{\partial E_{x}}
$$

leading to

$$
d_{x x x}=-2 Q_{11} P_{\mathrm{S}} \epsilon_{x x} \sin \alpha-2 Q_{12} P_{\mathrm{S}} \epsilon_{x y} \cos \alpha .
$$

Note that the first term indeed involves the divergent susceptibility component $\epsilon_{x x}$. The second term contains only the off-diagonal permittivity component $\epsilon_{x y}$, which for small values of the angle $\alpha$ reads $\epsilon_{x y} \approx-2 \epsilon_{11} \epsilon_{33} M \alpha$, and, therefore, this second term does not promise any anomalous property enhancement. Numerical calculation of the $d_{x x x}(\alpha)$ according to the Eq. (21) shows that the first term indeed reproduces the critical trend obtained from phase-field simulations. In fact, since domain B represents just one half of the sample 
volume, the macroscopic piezoelectric coefficient $d_{33}^{[100]}$ for large domains can be fairly well estimated as

$$
d_{33}^{[100]} \approx d_{11}^{\text {eff }}+0.5 d_{x x x},
$$

where $d_{11}^{\text {eff }}$ is taken from Eq. (13) and $d_{x x x}$ is taken from Eq. (21). The resulting theoretical dependence is shown by the dashed line in Fig. 6. The nice agreement of this simple theory with the phase-field simulations for large domain sizes demonstrates clearly that the bulk polarization-rotation mechanism described by Eqs. (15) and (21) is really responsible for the strongest piezoelectric enhancements simulated in this work as well as in the work of Ref. 13.

\section{CONCLUSION}

In summary, in this study we have used a phase-field approach to simulate piezoelectric response of domainengineered $\mathrm{BaTiO}_{3}$ single crystal with a regular array of mechanically compatible $90^{\circ}$ charged domain walls. Charged domain walls were stabilized by fixed planar interfacial charge located at their equilibrium positions. Assumed domain configuration consists of alternating $\mathrm{HH}$ and TT-type parallel boundaries. It was seen that both the domain period and the magnitude of the stabilizing interfacial charge (expressed through the dimensionless parameter $\rho^{*}$ ) influences the macroscopic piezoelectric response of such domain engineered material. It was found that the parameters $\rho^{*}$ and $w_{\mathrm{D}}$ also control quite sizable changes in the equilibrium field of polarization in the whole volume of the sample; mapping of the stable polarization configurations revealed stability regions of several qualitatively different states in the investigated range of these parameters (shown in Fig. 7). In fact, the strongest modification of the piezoelectric response occurs near boundaries of these stability regions.

Remarkably, we have seen that the longitudinal piezoelectric coefficient $d_{33}^{[100]}$ associated with the pseudocubic direction [100] can attain magnitudes of the order of $1000 \mathrm{pC} / \mathrm{N}$. Phase-field simulation revealed that this tremendous increase originates from the piezoelectric response of the normally piezoelectrically inactive domain state B (with nominal polarization perpendicular to the probed direction [100]). Complementary analytical calculations demonstrate that this enhancement is a consequence of the rotation of the spontaneous polarization within the domain B and the presence of free-energy minima associated with the energetically comparable metastable orthorhombic state. In addition, these calculations result in explicit formulas allowing to estimate this piezoelectric coefficient in similar materials with an equivalent GLD model (such as $\mathrm{KNbO}_{3}$ ).

It is likely that in a material with build-in charged layers of a convenient crystallographic orientation and sufficiently large $\rho^{*}$, the charged domain wall sequence will appear spontaneously, similarly as in our simulations. Practical aspects of the preparation of the desired charge-doped ferroelastic domain boundaries are, for the moment, far from being clear to us. However, it has been demonstrated that ionic diffusion can introduce sizable build-in electric fields ${ }^{30,31}$ and it has been also recognized that ferroelastic boundaries might to act as highways for ionic diffusion. ${ }^{32,33}$ Also the observed domain-size dependence of the enhancement of piezoelectric performance in $\mathrm{BaTiO}_{3}$ crystals reported for example in Ref. 34 suggests that a suitable field-cooling experimental protocol could eventually lead to a peculiar domain structure that involves charged domain walls. Formation of charged domain walls is probably more favorable at high temperatures when the nominal spontaneous polarization appearing in Eq. (6) is smaller and simultaneous ionic transport processes are more likely to occur. Still another possibility is to introduce in the material atomic planes of suitable aliovalent ions using controlled layer-by-layer epitaxy techniques, such as pulsed layer deposition or molecular beam epitaxy, similarly as it was proposed for stabilization of charged $180^{\circ}$ domain walls in Refs. 35 and 36.

In summary, we have explored here the piezoelectric properties of ferroelectric perovskites with nominally charged twin boundaries. The previous efforts to realize domain-engineered enhancement of the piezoelectric properties of $\mathrm{BaTiO}_{3}$ were mostly aimed to decrease the domain size. ${ }^{3}$ On the other hand, this study implies that variation of $\rho^{*}$ provides even more straightforward way to approach the critical polarization rotation angle. Moreover, this mechanism holds even for large domains. Therefore this work hints towards a very different possibility for domain engineering. It seems that here described prospects of the property enhancement by build-in charged layers are extremely challenging.

\section{ACKNOWLEDGMENTS}

The work was supported by the Sciex-NMS ${ }^{\text {ch }}$ program of the Swiss Federal Government (Project Code 11.116). Authors are grateful to A. K. Tagantsev for useful suggestions and discussions and very detailed and critical reading of the manuscript. The research leading to these results has received funding from the European Research Council under the EU 7th Framework Programme (FP7/2007-2013)/ERC Grant Agreement No. 268058.
${ }^{1}$ S. B. Seshadri, A. D. Prewitt, A. J. Studer, D. Damjanovic, and J. L. Jones, Appl. Phys. Lett. 102, 042911 (2013).

${ }^{2}$ D. Damjanovic, J. Am. Ceram. Soc. 88, 2663 (2005).

${ }^{3}$ S. Wada, K. Yako, K. Yokoo, H. Kakemoto, and T. Tsurumi, Ferroelectrics 334, 17 (2006).

${ }^{4}$ J. Hlinka, P. Ondrejkovic, and P. Marton, Nanotechnology 20, 105709 (2009).
${ }^{5}$ J. Fousek, D. B. Litvin, and L. E. Cross, J. Phys.: Condens. Matter 13, L33 (2001).

${ }^{6}$ J. Rödel, Mech. Mater. 39, 302 (2007).

${ }^{7}$ M. Guennou, H. Dammak, and M. Pham Thi, J. Appl. Phys. 104, 074102 (2008).

${ }^{8}$ R. Ahluwalia, T. Lookman, A. Saxena, and W. Cao, Phys. Rev. B 72, 014112 (2005). 
${ }^{9}$ T. Sluka, D. Damjanovic, A. Tagantsev, E. Colla, M. Mtebwa, and N. Setter, in Applications of Ferroelectrics (ISAF), 2010 IEEE International Symposium on Applications of Ferroelectrics (IEEE, Los Alatimos, CA, 2010), pp. 1-4.

${ }^{10}$ M. Y. Gureev, A. K. Tagantsev, and N. Setter, Phys. Rev. B 83, 184104 (2011).

${ }^{11}$ M. Y. Gureev, P. Mokry, A. K. Tagantsev, and N. Setter, Phys. Rev. B 86, 104104 (2012).

${ }^{12}$ T. Sluka, A. K. Tagantsev, D. Damjanovic, M. Gureev, and N. Setter, Nat. Commun. 3, 748 (2012).

${ }^{13}$ Z. Li, H. Wu, and W. Cao, J. Appl. Phys. 111, 024106 (2012).

${ }^{14}$ P. Marton, I. Rychetsky, and J. Hlinka, Phys. Rev. B 81, 144125 (2010).

${ }^{15}$ J. Hlinka and P. Marton, Phys. Rev. B 74, 104104 (2006).

${ }^{16}$ H.-L. Hu and L.-Q. Chen, J. Am. Ceram. Soc. 81, 492 (1998).

${ }^{17}$ S. Semenovskaya and A. G. Khachaturyan, J. Appl. Phys. 83, 5125 (1998).

${ }^{18}$ S. Nambu and D. A. Sagala, Phys. Rev. B 50, 5838 (1994).

${ }^{19}$ P. Marton and J. Hlinka, Phase Transitions 79, 467 (2006).

${ }^{20}$ A. Artemev, J. Slutsker, and A. L. Roytburd, IEEE Trans. Ultrason. Ferroelectr. Freq. Control 55, 963 (2008).

${ }^{21}$ J. Slutsker, A. Artemev, and A. Roytburd, Phys. Rev. Lett. 100, 087602 (2008); H.-L. Hu and L.-Q. Chen, Mater. Sci. Eng. A 238, 182 (1997).

${ }^{22}$ J. Hlinka, Ferroelectrics 349, 49 (2007).
${ }^{23}$ Y. Xiang, R. Zhang, and W. Cao, J. Appl. Phys. 106, 064102 (2009).

${ }^{24}$ J. Erhart and W. Cao, J. Appl. Phys. 86, 1073 (1999).

${ }^{25}$ Y. Ishibashi and M. Iwata, Jpn. J. Appl. Phys. 37, L985 (1998); K. Carl and K. H. Härdtl, Phys. Status Solidi A 8, 87 (1971); M. Davis, M. Budimir, D. Damjanovic, and N. Setter, J. Appl. Phys. 101, 054112 (2007); Y. Ni and A. G. Khachaturyan, Acta Mechanica Solida Sinica 25, 429 (2012).

${ }^{26}$ W. Cao and L. E. Cross, Phys. Rev. B 44, 5 (1991).

${ }^{27}$ S. Imanaga and K. Miura, Jpn. J. Appl. Phys. 51, 031502 (2012).

${ }^{28} \mathrm{R}$. Blinc and A. P. Levanyuk, Incommensurate Phases in Dielectrics, Vol. 1 (North-Holland, Amsterdam, 1986).

${ }^{29}$ J. Hlinka, J. Petzelt, B. Brezina, and R. Currat, Phys. Rev. B 66, 132302 (2002)

${ }^{30}$ V. D. Kugel and G. Rosenman, Appl. Phys. Lett. 62, 2902 (1993).

${ }^{31}$ G. Rosenman, V. D. Kugel, and D. Shur, Ferroelectrics 172, 7 (1995).

${ }^{32}$ W. T. Lee and E. K. H. Salje, Appl. Phys. Lett. 87, 143110 (2005).

${ }^{33}$ W. T. Lee, E. K. H. Salje, and U. Bismayer, Phase Transitions 76, 81 (2003).

${ }^{34}$ S. Wada and T. Tsurumi, Br. Ceram. Trans. 103, 93 (2004).

${ }^{35}$ X. Wu and D. Vanderbilt, Phys. Rev. B 73, 020103(R) (2006).

${ }^{36}$ E. D. Murray and D. Vanderbilt, Phys. Rev. B 79, 100102(R) (2009). 